\title{
Desarrollo del nuevo paradigma educativo. Más allá de la formación online: la formación en red
}

(Development of the new education paradigm. Beyond on-line training: net-worked training)

\author{
LuIS ÁNgEl FERNÁNDEZ HeRmanA \\ Director
}

IBAN GARcía CASALS

Coordinador de Proyectos

RAFA MARTÍN

Coordinador del Master en Comunicación Digital

Empresa: en.red.ando (http://enredando.com/)

RESUMEN: En este artículo tratamos de analizar en primer lugar las ventajas que ha supuesto la integración de las Nuevas Tecnologías, sobre todo Internet y el correo electrónico, en la educación a distancia. Es lo que a se ha llamado e-learning o formación online.

Sin embargo consideramos que la formación online no aprovecha todas las posibilidades de la Red. En este artículo señalamos los aspectos que el e-learning replica de la formación a distancia limitándose casi únicamente a utilizar las nuevas tecnologías para la transmisión de documentación y el contacto a través de correo electrónico o web.

Desde en.red.ando proponemos la formación en red como la nueva manera de formación a distancia aprovechando al máximo las posibilidades que nos ofrecen las Nuevas Tecnologías.

La formación en red proporciona riqueza de contenidos y una importante base de conocimiento, gracias a la comunicación, la interactividad y la participación de profesores y alumnos. Y esa base de conocimiento es básica e imprescindible para una formación de alta calidad.

En en.red.ando hemos desarrollado en.medi@, una tecnología de gestión de conocimiento en red que aplicamos al proceso de formación en red, en concreto al Master en Comunicación Digital de en.red.ando y a los cursos y seminarios de formación que ofrecemos.

Formación a distancia - Formación online - Formación en red - Nuevas Tecnologías - Internet Correo electrónico - Base de conocimiento 
Luis Ángel Fernández Hermana, Iban García Casals, Rafa Martín

ABSTRACT: In this article we try to analyse what advantages the New Information Technologies-especially Internet and e mail- have meant for the development of distance education. What it has been called e-learning or on-line training.

Nevertheless, we think that e-learning does not take full advantage of all possibilities that Internet offers. In this article we want to outline some aspects of on-line training, like how it mimics the classical distance training. This use of New Information Technologies is limited almost only to transfer documentation and contact-keeping via e mail or web.

From en.red.ando we propose "networked training" as a new form of distance training, extracting the rich possibilities that New Information Technologies offer.

Networked training generates rich content and a very important knowledge base, due to the communication, interactivity and participation among teachers and students. That knowledge base is essential for a high quality training.

In en.red.ando we have developed en.medi@, an on-line knowledge management technology that we apply to the process of networked training, and in particular, to the en.red.ando's Master in Digital Communication and to courses and training seminars that we offer.

Distance training - On-line training - Networked training - New Technologies - Internet - e-mail Knowledge base

\section{DESARROLLO DEL NUEVO PARADIGMA EDUCATIVO. MÁS ALLÁ DE LA FORMACION ONLINE: LA FORMACIÓN EN RED ${ }^{1}$}

Con la popularización y el desarrollo de Internet -sobre todo a partir del año 1995- surgió lo que no tardó en denominarse e-learning o formación online. Este tipo de formación tiene en el PC, en Internet y en el correo electrónico sus mejores y más ventajosas herramientas para que la educación a distancia sea mucho más eficaz de lo que conocíamos hasta esa fecha.

La comunicación a través de la Red y el correo electrónico nos ha permitido olvidar los envíos postales de apuntes, ejercicios, y la comunicación con los tutores y profesores a través de sesiones presenciales o consultas puntuales por teléfono. La transferencia de ficheros a través de la world wide web ha hecho más ágil el envío de los textos, y el correo electrónico ha facilitado en grado sumo la comunicación con el tutor y los profesores.

Sin embargo además de esas ventajas que ofrece la Red en lo que a comunicación se refiere, en aspectos que ya conocíamos de la educación a distancia -comunicación con profesores, envío de material-, los sistemas de formación online ofrecen importantes posibilidades que amplían y mejoran la calidad respecto a la presencial aportando importantes valores añadidos con los que no se contaba por razones evidentes de ausencia de los recursos tecnológicos.

A continuación señalamos esas ventajas a las que hacemos alusión y que ofrece la formación online:

\footnotetext{
Este estudio se basa en la investigación y experiencia de en.red.ando en el campo del e-learning conocimiento de plataformas, técnicas y metodologías- y el desarrollo de la plataforma en.campus a lo largo de los dos últimos años.
} 
Desarrollo del nuevo paradigma educativo. Más allá de la formación online: la formación en red

D Permite agrupar en un espacio virtual, a disposición de los alumnos, a expertos de las organizaciones y universidades más prestigiosas sin que la distancia geográfica sea un obstáculo.

D Permite, además, reunir alumnos procedentes de todo el mundo, con el único límite de la lengua de comunicación que se escoja. El aula llega a ser realmente multicultural.

D La formación online hace posible recoger los diferentes puntos de vista y realidades que convergen en el espacio virtual. La riqueza de contenidos del curso es incomparablemente alta. El encuentro de los alumnos se produce en un espacio virtual pero cada uno sigue viviendo una realidad propia del país donde se encuentre. Esto tiene un continuo reflejo en el día a día del proceso de formación y en el enriquecimiento del mismo.

\section{CRÍTICA A LA FORMACIÓN ONLINE}

Muy a menudo, el e-learning no aprovecha todos los recursos que la tecnología pone a su disposición, con el consiguiente deterioro del proceso de formación a distancia. Esto sucede, por ejemplo, cuando:

D Los contenidos son una simple transposición a Internet de los utilizados en la enseñanza presencial y vienen definidos de antemano.

De esta forma se limitan las posibilidades de Internet a su rapidez en el envío de la documentación: en vez de hacerlo por correo postal se envía por correo electrónico o se publican en una web.

D Los contenidos están estructurados para ser utilizados casi sin cambios durante mucho tiempo, como mucho con periódicas actualizaciones.

No se aprovechan las mejoras que pudieran incorporar los alumnos o los profesores en documentos que están en formatos tecnológicos muy flexibles, -material digital-, que permitirían variaciones y actualizaciones continuas.

D Los campus virtuales disponen de aulas y zonas de prácticas en los que la interacción entre alumnos y profesores es limitada (a través de correo electrónico, o foros).

La ausencia de metodologías que permitan registrar la comunicación en bases de conocimiento organizadas, buscables y diseminables conspira contra la eficaz del propio proceso de comunicación.

D Los alumnos reciben y consumen el material docente de manera unidireccional, con una metodología a la que deben adaptarse sí o sí.

No se potencia ni facilita la interacción con los alumnos-participantes. La clase sigue siendo de un profesor hacia los alumnos.

Todos tenemos opiniones interesantes, experiencias, observaciones, etc. Los alumnos también y debe proporcionarse el sistema para recoger todas 
Luis Ángel Fernández Hermana, Iban García Casals, Rafa Martín

estos comentarios e incluirlos en la mejora de los materiales, de la dinámica de la clase, de la riqueza del debate.

D Los alumnos aprenden estudiando en solitario, interactuando más o menos ocasionalmente con compañeros y profesores.

No se proporcionan las herramientas técnicas para el trabajo en grupo y la interacción entre participantes.

La participación y la interacción entre alumnos y profesores son claves en el desarrollo de la clase y la riqueza de contenidos que se genere.

D La estructura de la formación experimenta pocos cambios de una edición a otra.

No hay sistemas que permitan integrar las mejoras, las observaciones de los alumnos en los contenidos del curso o las experiencias acumuladas en los procesos de formación a través de la Red.

\section{UN PASO MÁS: LA FORMACIÓN EN RED}

En en.red.ando hemos desarrollado en.medi@, una tecnología de gestión de conocimiento en red que aplicamos al proceso de formación en red, en concreto al Master en Comunicación Digital de en.red.ando y a los cursos y seminarios de formación que ofrecemos. La tecnología en.medi@ brinda las siguientes posibilidades:

D Consiste de una plataforma digital, una metodología y una gente preparada para aplicarla. La plataforma está estructurada de tal manera que posibilite, por una parte, unos flujos de comunicación eficientes y, por la otra, consolidar una base de conocimiento a la medida de las necesidades de un curso de gran calidad.

La comunicación entre los alumnos y profesores participantes se desarrolla en dicha plataforma de una manera fácil, ágil y continua. Todas las interacciones quedan automáticamente clasificadas y sus resultados son diseminables.

El debate y los documentos que se generan quedan publicados en una plataforma web, registrados en base de datos para su indexación, y enviado a cada una de las cuentas de correo de cada participante suscrito. Lo que facilita el uso y la comunicación.

Esto propicia la participación e interacción. Y a su vez incide en la mejora y adaptación de los contenidos y en la creación de una base de conocimiento de una calidad óptima sobre unos objetivos marcados por el curso.

D La base de la formación en red es la comunicación y la creación y gestión de un contenido que crece y aumenta con la participación y la interacción de los usuarios de la plataforma. En el caso de un Master, gracias a la interacción de estudiantes, profesores, tutores y consultores. 
Desarrollo del nuevo paradigma educativo. Más allá de la formación online: la formación en red

Da formación se lleva a cabo con y en Internet, utilizando desde el principio herramientas digitales, aplicando las posibilidades más avanzadas en comunicación digital. De esta forma los alumnos desarrollan las habilidades propias y necesarias en el uso de unas herramientas básicas y absolutamente necesarias en cualquier medio laboral.

D La incorporación a cada curso de la base de conocimiento generada en la edición anterior permite ofrecer un producto mucho más acorde con los cambios de la realidad profesional, de las empresas y del mercado de trabajo. Así, los contenidos se van afinando en función de las demandas reales de los alumnos y del contexto socio-económico.

D La producción de contenido y conocimiento nuevo y original, ya sea por parte de los alumnos o por parte de los profesores está disponible de forma inmediata y redunda en una formación actual y de máxima calidad.

- Esta riqueza cristaliza en la creación de documentación y contenidos de alta calidad referidos a un campo de conocimiento en concreto. De esta forma, la clase no termina cuando ésta llega a su fin: los documentos y los conocimientos generados quedan almacenados digitalmente y son consultables en todo momento, incluso desde cualquier parte del mundo, con la única condición de estar conectado a la Red.

Desde en.red.ando pensamos que la formación online debe ser más flexible y estar basada en la participación e interacción de los participantes en un curso. Hablaríamos entonces de lo que denominamos formación en red. Cada una de las personas participantes y distribuidas por la estructura red del curso tiene información y experiencias que, puestas en común, dan lugar a un conocimiento compartido, que debe estar a disposición de todos, una vez organizado, clasificado, estructurado y con su correspondiente motor de búsqueda.

Cualquier proceso de "e-learning" que no aproveche las ventajas que ofrece la red, estará en realidad reproduciendo viejos métodos y rebajando las posibilidades formativas actuales.

\section{EN.MEDI@: METODOLOGÍA PARA LA FORMACIÓN EN RED}

\section{EL SISTEMA}

en.medi@ es una tecnología diseñada para generar conocimiento mediante la participación e interacción de los alumnos inscritos al curso en torno a unos objetivos de trabajo previamente establecidos en el currículum del mismo.

en.medi@ consta de:una plataforma digital programable, una metodología y gente preparada para aplicarla (moderador y gestores del conocimiento). 
Los participantes intervienen en el espacio virtual simultáneamente como productores y consumidores de información y conocimiento. Toda la actividad se rige por unas pautas metodológicas impartidas por un moderador.

El espacio está asistido por uno o más Gestores del Conocimiento en Red que aportan documentos y materiales para nutrir el debate. Estas labores de gestión de conocimiento en red las pueden desarrollar tanto los propios alumnos (tras un período de aprendizaje), los profesores, o también un equipo de profesionales dedicados a tal tarea. El objetivo es ahondar los temas que se están tratando en el curso y tratar de llegar a una especie de "estado de la cuestión".

El resultado es la producción de información y conocimiento de alta calidad sujeta a los objetivos fijados.

Esta dinámica genera productos originales que incrementan la inteligencia existente en el espacio y mejoran los contenidos para posteriores ediciones del mismo curso.

enmedi@ es, por tanto, un captador de capital intelectual. Lo hace emerger de manera visible a través de un diseño específico de los flujos de comunicación, del trabajo en red y del papel de los Gestores de Conocimiento en red del espacio.

El capital intelectual generado queda automáticamente ordenado y almacenado en bases de datos, con herramientas de recuperación que permiten consultarlo de una manera fácil y rápida. Generar esta base de conocimiento es el objetivo de la tecnología en.medi@. Y esa base de conocimiento es básica e imprescindible para una formación de alta calidad.

\section{AROUITECTURA TECNOLÓGICA DE EN.MEDI@}

Los espacios virtuales creados con la tecnología desarrollada por en.red.ando son accesibles al usuario mediante el simple manejo de la web y del correo electrónico. No es necesario descargar ningún programa, ni plataformas propietarias, ni herramientas que no sean aquellas que se utilizan habitualmente cuando uno se conecta a Internet. Por ello, cualquier persona puede acceder a en.medi@ y utilizar sus recursos sin necesidad de descargar o instalar en su ordenador ningún tipo de software adicional.

La plataforma virtual sobre la que descansa en.medi@ está programada para que sea una parte más de Internet, aunque una parte donde todo sucede "del revés" de como sucede en Internet: la información y el conocimiento están estructurados, la navegación está orientada por los objetivos que organizan el espacio y los usuarios intercambian y encuentran información pertinente para sus intereses. De hecho, se constituyen en una Red Inteligente orientada a la formación en red. 
Desarrollo del nuevo paradigma educativo. Más allá de la formación online: la formación en red

PERFIL ACADÉMICO Y PROFESIONAL DE LOS AUTORES

Luis Ángel Fernández Hermana (luisangel@enredando.com) es Director de en.red.ando consultor de Nuevas Tecnologías de la Información, periodista, y el creador de la tecnología en.medi@

Iban García Casals (iban@enredando.com) es Coordinador de Proyectos de en.red.ando. Periodista y consultor de comunicación. Se ocupa del desarrollo y ejecución de los proyectos basados en la tecnología en.medi@ en empresas e instituciones.

Rafa Martín (rafa@enredando.com) es coordinador del Master en Comunicación Digital de en.red.ando. Desde el año 2000 se ocupa de coordinar cursos de formación online y el Master en Comunicación Digital de en.red.ando que acredita la Universidad de Vic. 